\title{
Effective Capacity of Imperfect Adaptive Wireless Communication Systems
}

\author{
Ulrico Celentano \\ Centre for Wireless Communications \\ P.O. Box 4500, FIN-90014, University of Oulu, Finland \\ Tel: + 35885532865 , Fax: + 35885532845 \\ Email: ulrico.celentano@ee.oulu.fi
}

\author{
Savo Glisic \\ Telecommunication Laboratory \\ P.O. Box 4500, FIN-90014, University of Oulu, Finland \\ Tel.: + 3588553 2852, Fax: + 35885537615 \\ Email: savo.glisic@ee.oulu.fi
}

\begin{abstract}
This paper presents a model for the link service capacity that an imperfect adaptive radio link provides to upper layers. As the main contribution of this paper, the model includes a number of imperfections in the link adaptation chain, as well as implementation implications. The average goodput is expressed also in compact form and its dependence on the impairments is discussed using analytical, numerical, and simulations results. The model, to be used for analyses at upper layers, integrates physical channel, transceiver characteristics, and imperfections in a flexible way, by independent, separate matrices.
\end{abstract}

\section{INTRODUCTION}

Wireless systems are subject to varying channel conditions, due to propagation properties of the environment and possibly mobility of terminals. Transceivers structures themselves dynamically change by using physical layer techniques, referred to as PHY, introduced to compensate for those impairments.

The model discussed in this paper includes physical channel and the PHY. The same portion of the protocol stack is covered in a link layer model, called effective capacity link model [1], which models directly few link layer parameters used in queuing analysis, without including imperfections of the physical layer or adaptation in link layer. A similar definition of MAC channel is given in [2], where a model for packet losses is included, taking into account physical channel, modulation and channel coding, and some other functions of the data link layer, but only for transceivers with fixed structure. Performance of adaptive radio links has been studied in presence of AWGN channel [3], or fading channels but without channel coding [4], or for coded systems with specific channel coding schemes and decoding methods [5] [6].

The final expression for the average throughput is given in (11). In this expression, physical channel and transceiver characteristics, as well as imperfections, are included. This model is then used in illustrative examples. Rather than being a performance analysis of a specific system, the purpose is to show how imperfections impact on system performance and the importance to include those into the model.

\section{The MAC Channel Model}

The service capacity depends on the gross transmission bit rate and the errors at the receiver. In fixed-PHY systems, the error rate absorbs the variability of the channel. Conversely, with link adaptation, the PHY mode from the set $\mathcal{M}$ is chosen so that the error rate is kept bounded, whereas the bit rate changes with the state. Our model describes the dynamics of those metrics, including the physical channel and the PHY characteristics, as well as imperfections in the link adaptation (Fig. 1). This is referred to as MAC channel model.

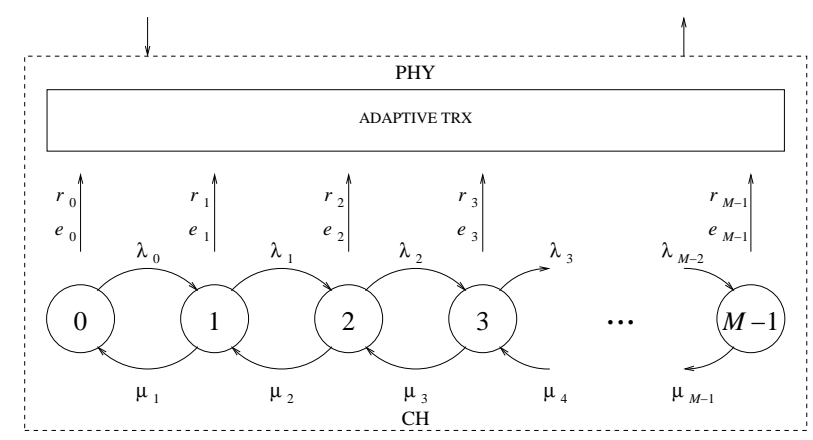

Fig. 1. The MAC channel model.

Channel conditions may be represented, e.g., by the signal to interference-plus-noise ratio (SINR), but in the following, we will denote with $\gamma$ a generic metric of the link quality. In the model considered in this paper, the true quality level is modeled as a continuous time Markov chain (CTMC), instead of discrete time model sometimes used for symbol or packet error models [7] [8]. In our model, state transitions are associated with boundary crossings of the metric.

The goal of this study is to represent the behavior of the signal quality level in terms of presence in a region, and to link it to the service offered by the PHY, with a granularity given by the cardinality of the modes set, $M=|\mathcal{M}|$, which is typically small in real systems. For example, the number of PHY modes is seven or eight in HSDPA, IEEE 802.11a, and ETSI HiperLAN/2, and up to five in IEEE 802.16a. When $M$ is small, the model exhibits lower frequencies due to practical constraints (Sect. III). The framework presented in this paper is intended to be applicable to generic channel processes that are continuous, and that can be represented by a small number of states. If a stochastic process that assumes values on a continuous set $\Gamma \subseteq \mathbb{R}$ split in a finite number of contiguous, non-overlapping intervals, is continuous, then the derived CTMC is a birth-death process [9]. 
With state $S_{i}$ associated with $\gamma \in\left[\gamma_{i}, \gamma_{i+1}\right)$, see (3), the generic elements of the state transition rate matrix, $\mathbf{Q}$ can be written as

$$
q_{k, k+1}=p(k, k+1 ; t, t+\Delta t) / \Delta t=N_{k+1}^{+} / \pi_{k},
$$

where $N_{k}^{+}$is the expected number of times level $k$ is crossed upwards in a second, the level crossing rate (LCR), and $\pi_{k}$ is the probability of state $S_{k}$. Similarly

$$
q_{k, k-1}=p(k, k-1 ; t, t+\Delta t) / \Delta t=N_{k}^{-} / \pi_{k} .
$$

If switching hysteresis is adopted, definition of LCRs must be properly modified using the modified levels (Sect.III) [9]. Quantities in (1) and (2) can also be evaluated from observation of measurement traces or from simulations of complicated systems.

\section{Model OF Goodput}

The error rate is a fundamental performance measure for quality of service (QoS). According to QoS requirements (target error rates), the domain of the signal quality metric is divided into $M$ contiguous, non overlapping intervals. Each region is associated with a state of the CTMC, and each of the $M$ PHY modes is associated with a region of the quality metric. Each state is therefore characterized by its bit rate and error rate: $S_{i} \Rightarrow M_{i} \Rightarrow r_{i}, e_{i}$. The set of the threshold levels, i.e., the mode switching points, is defined so that:

$$
\begin{aligned}
\gamma \in\left[\gamma_{i}, \gamma_{i+1}\right) & \Rightarrow S_{i} \Rightarrow M_{i}, 0<i \leq M-1 \\
\gamma \in\left(\gamma_{0}, \gamma_{1}\right) & \Rightarrow S_{0} \Rightarrow M_{0},
\end{aligned}
$$

where $\gamma_{0}=-\infty$ and $\gamma_{M}=+\infty$. The nominal value of the metric in each region can be defined as the average value or the center value in the range. Switching points can be defined also using other methods, including optimization methods [10]. The model described in this paper is independent of the method used to define the set $\mathcal{S}$.

To avoid too frequent mode switching, switching hysteresis can be introduced by fixing distinct values for falling, $\gamma^{-}$, and rising, $\gamma^{+}$, thresholds: $\gamma_{i}^{ \pm}=\gamma_{i} \pm \varphi_{i}^{ \pm}, \varphi_{i}^{ \pm} \geq 0$. With $\varphi_{i}^{+}=\varphi_{i}^{-}=\varphi, 2 \varphi$ is the width of the hysteresis region. Alternatively, switching thresholds can be selected according to the following equation:

$$
\int_{\gamma_{i}-\varphi_{i}^{-}}^{\gamma_{i}} p_{\gamma}(\gamma) \mathrm{d} \gamma=\int_{\gamma_{i}}^{\gamma_{i}+\varphi_{i}^{+}} p_{\gamma}(\gamma) \mathrm{d} \gamma
$$

At link layer, a packet is considered valid if no error is present or all errors have been corrected. The entity of the packet error rate (PER) depends on the error correction coding gain (Sect.V). Distinct channel coding schemes may be adopted for header and payload [9]. We assume in the following that the same channel coding scheme is applied to both parts.

Because of channel coding redundancy and packet header, the informative bit rate is smaller than the raw bit rate. The informative bit rate for a given PHY mode (still including residual errors) can be written as $r_{i}=\eta_{h} \eta_{c, i} k_{i} / T_{s}$, where $\eta_{h}=L_{i} / L_{d}$ is the header efficiency, $\eta_{c}=L_{d} /\left(L_{d}+L_{o}\right)$ is the channel coding efficiency (code rate) including redundancy $L_{o}, L_{d}=L_{i}+L_{h}$ is the total size of payload plus header, $k_{i}$ is the number of bits per symbol of the $i$-th mode modulation scheme, and $T_{s}$ is the symbol duration.

The link service capacity, denoted with $R_{c}(t ; \zeta)$, is the rate at which error free information units are transmitted through the channel, and is referred to as goodput. We start from a similar expression for the link service rate as given in [11] for ideal systems with fixed PHY. We extend it first to time varying and adaptive systems, (5). Finally, we work further the new expression to introduce imperfections, (10) and (11).

The throughput vector $\mathbf{r}=\left\{r_{i}\right\} \in \mathbb{R}^{M}$ is defined as $\mathbf{r}^{\top} \doteq\left[\begin{array}{lll}r_{0} & \cdots & r_{M-1}\end{array}\right]$ with $r_{i}$ defined above. The normalized goodput matrix $\mathbf{Y}=\left\{y_{i j}\right\} \in \mathbb{R}^{M \times M}$ is defined as $\mathbf{Y}=$ $\left\{y_{i j} \doteq 1-e_{i j}\right\}$, where $e_{i j}$ denotes the residual error rate when the mode $M_{i}$ is used and the channel is in effective state $S_{j}$ (see Sect. IV). The residual error rate after reassembling $e$ has the same value of the PER: $e=p_{E}$. The elements of the state probability vector $\boldsymbol{\pi}, \boldsymbol{\pi}^{\boldsymbol{\top}} \doteq\left[\begin{array}{lll}\pi_{0} & \cdots & \pi_{M-1}\end{array}\right]$, are the probabilities of quality regions $\mathcal{S}$.

If the adaptive system timely follows without errors the channel state, our expression for the average goodput is

$$
\left.\bar{R}_{c}\right|_{\text {ideal }}=\sum_{i=0}^{M-1} r_{i}\left(1-e_{i i}\right) \pi_{i}=\mathbf{r}^{\top} \operatorname{diag}(\mathbf{Y \Pi}),
$$

where $\boldsymbol{\Pi}=\operatorname{diag}(\boldsymbol{\pi})$ is the diagonal matrix having as diagonal elements the elements of vector $\boldsymbol{\pi}$, and $\operatorname{diag}(\mathbf{A}) \doteq\left[a_{i i}\right]$ is the operator that extracts the vector of diagonal elements of matrix A.

\section{MOdel OF IMPAIRMENTS}

In (5), due to perfect set-up of the transceiver configuration, only diagonal values of matrix $\mathbf{Y}$ are used, since the $i$-th mode $M_{i}$ is always used when the channel is in $S_{i}$. In case of erroneous mode selection, the gross bit rate depends on the used mode whereas the error rate depends on both the used mode and the effective state. To cover this case, (5) must be generalized.

The signal commanding mode switching can be defined by receiver controlled closed loop, or by transmitter controlled open loop. The mode information, in general affected by error, is sent in the (feedback) communication channel, and acquired corrupted and with delay. In practice, it is impossible for the transmitter to know what the actual channel state will be at the reception instant and site.

The parameter used for the mode switching can be expressed as $\hat{\gamma}(t)=\gamma\left(t-\tau_{e}\right)+\varepsilon_{e}+\varepsilon_{f}$, where $\gamma$ and $\hat{\gamma}$ are the true and the estimated value, respectively, of the metric, $\varepsilon_{e}$ is the estimate error, $\varepsilon_{f}$ is the feedback error, and $\tau_{e}$ is the estimation delay. Among these three terms, the first has more important effect in the open loop case, whereas in the closed loop case, the other two terms are dominant.

All these imperfections in the adaptation chain are taken into account in the proposed model described in this paper. 
The actual properties of the related errors depend on the particular system configuration. In this paper, we are not interested in studying a specific system, but rather we want to model common sources of errors in a flexible way open to generalizations.

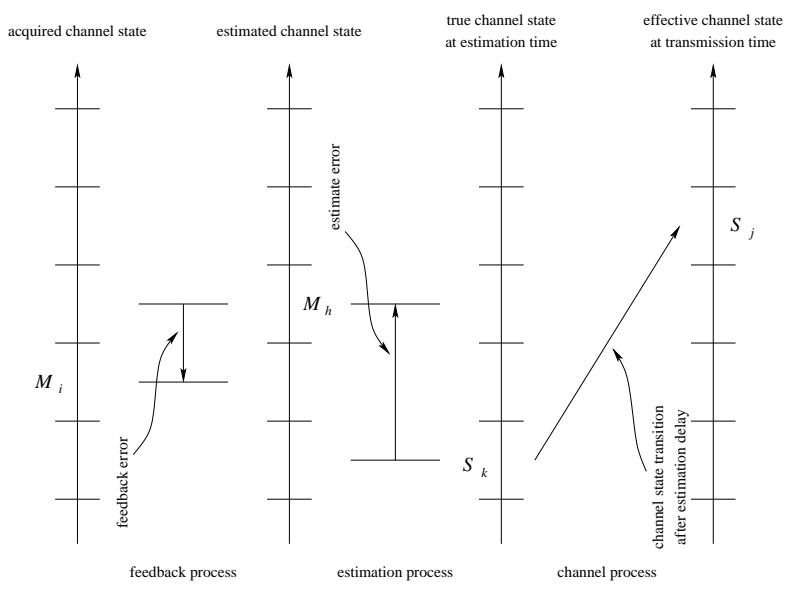

Fig. 2. Model of imperfections.

The model of imperfections is depicted in Fig. 2. At estimation time $t$, the true channel quality metric falls in the $k$-th region, say $S(t)=k$, and the metric is estimated. Based on this estimate $S(t)=h$, the mode is selected. The mode is transmitted in the feedback channel subject to errors, and $M_{i}$ is acquired at the transmitter (or at the receiver, in the open loop case). This mode is used for transmission (closed loop) (or reception, open loop). In the mean time, the effective channel quality metric falls in general in a new region, $S\left(t+\tau_{e}\right)=j$, due to possible channel state transitions during the estimation delay $\tau_{e}$. The final effect of the entire process is that in general mode $M_{i}$ is used at transmission time, when the channel is in effective state $S_{j}$. The probability of this event can be written as $P\left(M_{i}, M_{h}, S_{k}, S_{j}\right)$. Assuming the estimation process and the channel process independent, and assuming that the feedback channel is independent of the direct channel, we can write

$$
\begin{aligned}
& P\left(M_{h}, S_{k}, M_{i}, S_{j}\right)= \\
& =P\left(S_{k}\right) P\left(M_{h} \mid S_{k}\right) P\left(M_{i} \mid M_{h}\right) P\left(S_{j} \mid S_{k}\right) .
\end{aligned}
$$

The independence of direct and feedback channel relates to duplexing scheme, MAC frame structure, terminal speed, etc. The independence of estimation and channel process related mainly to the estimation algorithm.

The three last terms in (6) are treated separately in the sequel.

The metric estimate may be erroneous. The mode estimation probability matrix $\mathbf{H}^{(e)}=\left\{h_{h k}^{(e)}\right\} \in \mathbb{R}^{M \times M}$ models the effects of the imperfections in the estimation process due to noise. Its generic element $h_{h k}^{(e)}$ represents the probability of selecting mode $M_{h}$ with the true channel in state $S_{k}$ at estimation time:

$$
\mathbf{H}^{(e)}=\left\{h_{h k}^{(e)} \doteq \operatorname{Pr}\left\{M_{h} \mid S_{k}\right\}\right\} .
$$

Equation (7) represents the second term in (6).

The channel state may change during the estimation delay $\tau_{e}$. The delayed channel transition matrix $\mathbf{H}^{(d)}=\left\{h_{k j}^{(d)}\right\} \in$ $\mathbb{R}^{M \times M}$ models the effects of estimation delay. Its generic element $h_{k j}^{(d)}$ represents the probability that given the true channel was in state $k$ at estimation time, at transmission time the channel is in state $j$ :

$$
\mathbf{H}^{(d)}=\left\{h_{k j}^{(d)} \doteq \operatorname{Pr}\left\{S\left(t+\tau_{e}\right)=j \mid S(t)=k\right\}\right\} .
$$

Equation (8) represents the last term in (6).

The code representing the PHY mode or, equivalently, the related signal quality region, may get corrupted during its transmission through the feedback channel. The mode acquisition probability matrix $\mathbf{H}^{(f)}=\left\{h_{i h}^{(f)}\right\} \in \mathbb{R}^{M \times M}$ models the probability of mode error due to errors in the feedback channel and has generic element given by:

$$
\mathbf{H}^{(f)}=\left\{h_{i h}^{(f)} \doteq \operatorname{Pr}\left\{M_{i} \mid M_{h}\right\}\right\} .
$$

Equation (9) represents the third term in (6).

Combining (5) and (6), and remembering the model in Fig. 2 , we get the expression of the average goodput, i.e., the link service rate in presence of imperfections:

$$
\bar{R}_{c}=\sum_{i=0}^{M-1} h_{i i}^{(f)} r_{i} \sum_{j=0}^{M-1}\left(1-e_{i j}\right) \sum_{k=0}^{M-1} h_{i k}^{(e)} \pi_{k} h_{k j}^{(d)} .
$$

It is easy to see that the average goodput in (10) can be written in compact form as

$$
\bar{R}_{c}=\tilde{\mathbf{r}}^{\top} \operatorname{diag}\left(\mathbf{Y} \Theta^{\top}\right),
$$

where $\tilde{\mathbf{r}} \doteq\left\{h_{i i}^{(f)} r_{i}\right\}=\mathbf{r} \odot \operatorname{diag} \mathbf{H}^{(f)}$ and $\boldsymbol{\Theta}=\left\{\vartheta_{n j}\right\} \in$ $\mathbb{R}^{M \times M}$ is the mode-channel probability matrix, $\Theta \doteq$ $\mathbf{H}^{(e)} \boldsymbol{\Pi} \mathbf{H}^{(d)}$, whose generic element models the probability of using mode $M_{n}$ with the channel in the effective state $S_{j}$.

The scalar normalized average goodput corresponds to the average goodput of a system adopting an hypothetical set of PHY modes all having unitary transmission rate:

$$
\bar{R}_{r}=\left\|\operatorname{diag}\left(\mathbf{Y} \Theta^{\top}\right)\right\|_{1} .
$$

The use of this quantity will be clear in the analysis.

In the ideal case of perfect link adaptation, $\mathbf{H}^{(e)}=\mathbf{H}^{(d)}=$ $\mathbf{H}^{(f)}=\mathbf{I}$, and (11) reduces to (5). Single PHY mode systems are considered in this model as a special case with $\mathbf{H}^{(f)}=\mathbf{I}$, $\mathbf{H}^{(d)}=\mathbf{I}$, and static mode selection leading to a matrix $\mathbf{H}^{(e)}$ having as non-zero elements all 1 in the $i$-th row, if mode $M_{i}$ is implemented in the system: $h_{h k}^{(e)}=\delta_{h i} \delta_{k k}$, where $\delta_{m n}$ is the Kronecker delta.

\section{ILLUSTRATIVE EXAMPLES}

For illustration purposes, we apply the above analysis tool to the study of a sample system and investigate its sensitivity to imperfections and implementation constraints.

The set of PHY modes includes a no-transmission mode for values of the signal quality that do not comply with minimum 
service requirements. The modulation schemes used in the numerical analysis are BPSK, QPSK, 8QAM, and 16QAM. The channel coding scheme is the half rate convolutional code $\left(g_{0}=133_{8}, g_{1}=171_{8}, K=7\right)$. Coding gain curves are obtained by fitting known values [9]. With independent bit errors at link layer (e.g., by sufficient interleaving), the PER is given by $p_{E}=1-\left[1-p_{e}\left(\gamma G_{c}(\gamma)\right)\right]^{L_{p}}$, where $p_{e}(\gamma)$ is the bit error rate of the uncoded system, $G_{c}(\gamma)$ is the coding gain, $\gamma$ is the SINR per bit, and $L_{p}$ is the packet length in bits. Any channel coding scheme whose coding gain curve $G_{c}(\gamma)$ is known can be included in this model. Switching thresholds are obtained from a required PER of $10^{-5}$ for a packet length of 2048 bits (Sect. III). Although both multipath fading and shadowing are generally superimposed, only one at a time affects link adaptation [5]. We assume Ricean fading process, line-of-sight component with zero Doppler frequency, uncorrelated Gaussian noise components, and Jakes shaped Doppler power spectral density or isotropic scattering.

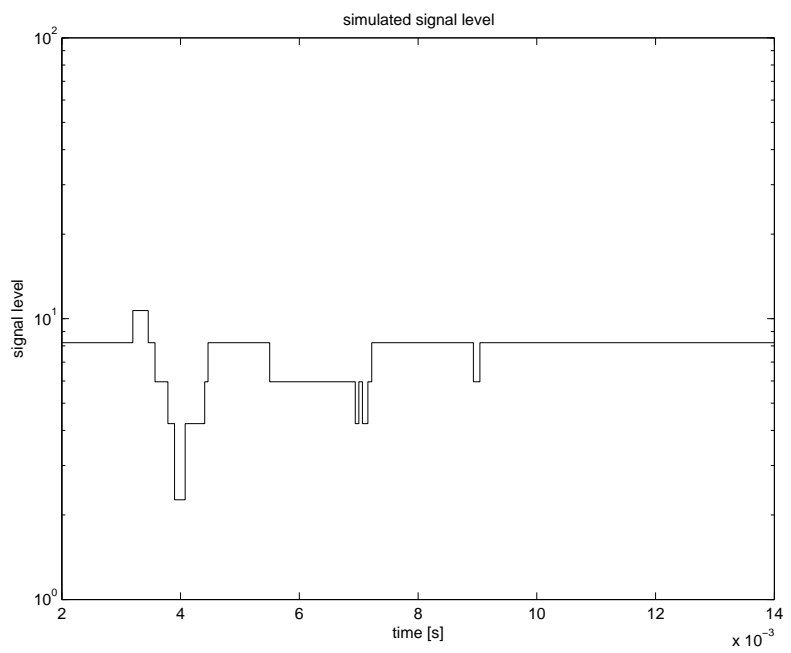

Fig. 3. Snapshot of CTMC simulations.

Fig. 3 shows a snapshot of the time series obtained by simulations with our CTMC model with $f_{\max }=300 \mathrm{~Hz}$. It can be seen that large changes in the metric, rapid changes, and relatively long steady periods are realizable. The model is able to reproduce values in a wide range [9].

The expressions for the state probabilities after introducing the hysteresis derived in [9], specialized for the case of equal and symmetric hysteresis regions, and neglecting higher order infinitesimals, are

$$
\pi_{i}^{(h)} \approx \pi_{i}+\Delta_{C D F}\left(2 \pi_{i}-\pi_{i+1}-\pi_{i-1}\right),
$$

where $\pi_{i}^{(h)}$ and $\pi_{i}$ are the state probabilities with and without hysteresis, respectively, and $\Delta_{C D F}$ is the half width of the hysteresis region. Fig. 4 shows the sensitivity of state probabilities to $\Delta_{C D F}$ in this latter case, with regions defined as in (4). Bars are simulated values, whereas points are the theoretical values given by (13). For larger values of $\Delta_{C D F}$, states with larger probability, the central ones in our case, have their probability

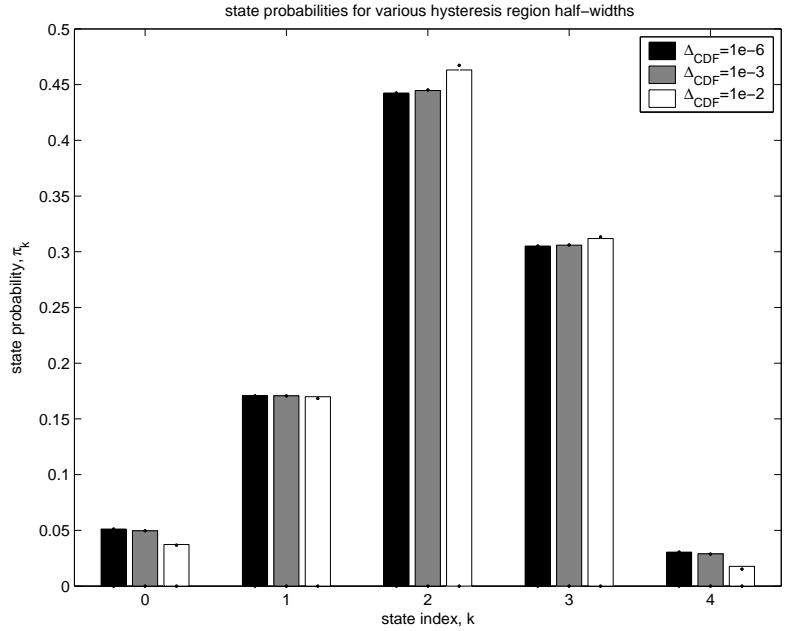

Fig. 4. Sensitivity of state probabilities to $\Delta_{C D F}$.

further increased by the introduction of hysteresis. It can be observed that up to about $\Delta_{C D F}=10^{-3}$, state probabilities are almost unaffected.

The sensitivity of the average goodput $\bar{R}_{c}$ to variance of channel estimation error $\sigma_{e}^{2}$ is studied assuming absence of estimation delay and error free feedback channel. The distribution of the estimation error depends on the specific estimation technique. Analytical and/or empirical distributions for estimation error are generally unknown [5]. For this illustrative example we assume the estimation error added to the true value to be Gaussian distributed. Under these assumptions, it is easy to see that the generic element of matrix $\mathbf{H}^{(e)}$ defined in Sect. IV can be written as

$$
h_{h k}^{(e)}=\frac{1}{2}\left[\operatorname{erfc}\left(\frac{\xi_{h}^{\prime}-\bar{\xi}_{k}}{\sigma_{e}}\right)-\operatorname{erfc}\left(\frac{\xi_{h}^{\prime \prime}-\bar{\xi}_{k}}{\sigma_{e}}\right)\right],
$$

where $\operatorname{erfc}(\cdot)$ is the Gaussian complementary error function, $\sigma_{e}$ is the standard deviation of the estimation error, $\bar{\xi}_{k}$ is the nominal value of the metric in the $k$-th interval $S_{k}$, and $\xi_{h}^{\prime}$ and $\xi_{h}^{\prime \prime}$ are the lower and upper boundaries, respectively, of $S_{h}$.

Fig. 5 shows the comparison of simulation (crosses) and theoretical results (lines). The curve of the average goodput $\bar{R}_{c}$ (solid line) exhibits a maximum for variance larger than zero. This behavior is explained by the fact that, in the expression of $\bar{R}_{c}$, the averaging is weighted by the gross bit rate. The scalar normalized average goodput $\bar{R}_{r}$ (dashed line), defined in (12), takes into account only the effects of the error rate and is independent of the transmit gross bit rate. As expected, the curve of $\bar{R}_{r}$ is monotonically decreasing.

In order to simplify the analysis, we assume that the estimation delay is negligible compared to the minimum intertransition time (time between state changes). For our analysis it is sufficient to assume that, $\forall i, \lambda_{i} \tau_{e}=q_{i, i+1} \tau_{e} \ll 1$ and $\mu_{i} \tau_{e}=q_{i, i-1} \tau_{e} \ll 1$, where $M$ is the cardinality of sets of PHY modes $\mathcal{M}$ and quality regions $\mathcal{S}$. Under these assumptions, at most one state change can occur during $\tau_{e}$. It is straightforward to see that $\mathbf{H}^{(d)}=\mathbf{I}+\mathbf{Q} \tau_{e}$. 


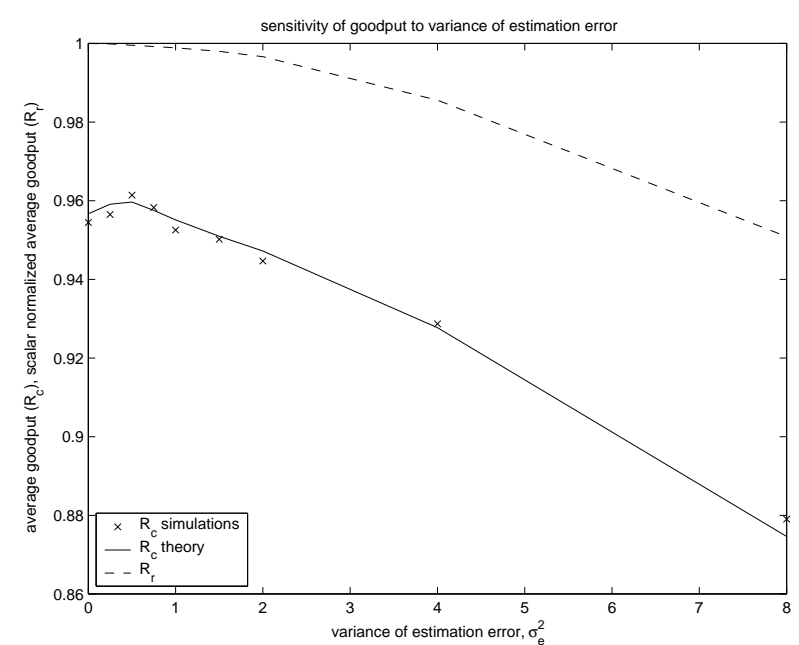

Fig. 5. Sensitivity of $\bar{R}_{c}$ to $\sigma_{e}^{2}$.

Considering perfect estimation and errorless feedback channel, and under the previous assumptions for the estimation delay model, the average goodput is expressed by:

$$
\bar{R}_{c}\left(\tau_{e}\right)=\mathbf{r}^{\top} \operatorname{diag}\left(\mathbf{Y \Theta}^{\top}\right)=\bar{R}_{c}(0)+\tau_{e} \Psi_{d}
$$

with $\bar{R}_{c}(0)$ given by (5) and where

$$
\Psi_{d}=\frac{\partial \bar{R}_{c}}{\partial \tau_{e}}=\mathbf{r}^{\top} \operatorname{diag}\left(\mathbf{Y} \mathbf{Q}^{\top} \mathbf{\Pi}\right)
$$

is the unitary drift of the average goodput from the ideal conditions due to the estimation delay. Numerical results match the theoretical expression in (15) [9].

To identify a PHY mode in $\mathcal{M}$, a control message with $\left\lceil\log _{2} M\right\rceil$ bits is used. The distance in bits among all pairs of codewords is given by the elements of a symmetric matrix having null elements on the diagonal, and defined by

$$
\mathbf{D}=\left\{d_{i j} \doteq \sum_{n=1}^{\left\lceil\log _{2} M\right\rceil} w_{i}^{(n)} \otimes w_{j}^{(n)}\right\}
$$

where $w_{i}^{(n)}$ is the $n$-th bit of the $i$-th codeword and $\otimes$ denotes the modulo 2 bit-wise product of the codewords. The element of matrix $\mathbf{H}^{(f)}$ can be written as

$$
h_{i h}^{(f)}=p_{e, r, f}^{d_{i h}}\left(1-p_{e, r, f}\right)^{\left\lceil\log _{2} M\right\rceil-d_{i h}},
$$

where $p_{e, r, f}$ is the residual bit error probability in the feedback channel, given by $p_{e, r, f}=p_{e}^{(f)}\left(\gamma G_{c}(\gamma)\right)$.

One way of coding mode identifiers is adopting the Gray code. Assume that the message is transmitted always using the strongest mode. Under the assumption of symmetric channel and with the error probability depending on the actual channel state, it has been seen that the effect of feedback errors is negligible. In fact, if the BER in the feedback channel $p_{e}^{(f)}$ is assumed fixed and independent of the state of the direct channel, the impact on $\bar{R}_{c}$ is no longer negligible only for values $p_{e}^{(f)}>10^{-2}$, so large to be out of range in adaptive systems and often in communications systems in general [9].

\section{Conclusions}

In this paper, a model for the link layer service capacity (average goodput) of adaptive radio link systems has been presented. The model includes the overall behavior of channel and imperfect link layer adaptive techniques in a flexible way open to generalizations.

The average goodput is expressed in a compact form including the impact of some imperfections in the adaptation chain (estimate error, feedback errors, and estimation delay) and implementation losses (introduction of hysteresis in the switching thresholds).

The characteristics of the system are independently represented by separate matrices, for the channel ( $\mathbf{Q}$ and $\boldsymbol{\pi}$ ), transmit rate (r) and error rates $(\mathbf{Y})$ for the given transceiver modes. Matrices for the probabilities of erroneous set-up due to estimation errors $\left(\mathbf{H}^{(e)}\right)$, estimation delay $\left(\mathbf{H}^{(d)}\right)$, and feedback errors $\left(\mathbf{H}^{(f)}\right)$ are also included. Due to the modular structure of the model, the analysis can be extended to more general and possibly complicated systems than the one used in the example.

The presented model can be effectively integrated in some analytical models for the analysis of the higher network layers.

\section{ACKNOWLEDGMENT}

This research was supported by the National Technology Agency of Finland, Nokia, the Finnish Defence Forces, Elektrobit and Instrumentointi.

\section{REFERENCES}

[1] D. Wu, and R. Negi, "Effective capacity: A wireless link model for support of quality of service," IEEE Trans. Wireless Commun., vol. 2, no. 4, pp. 630-643, July 2003.

[2] G. Liebl, T. Stockhammer, and F. Burkert, "Modelling and simulation of wireless packet erasure channels", Proc. Tech/MRPG Symp. Wireless Personal Commun., Blacksburg, VA, USA, pp. 203-214, May 2000.

[3] D. Qiao, and S. Choi, "Goodput enhancement in IEEE802.11a wireless LAN via link adaptation," Proc. IEEE Int. Conf. Commun. (ICC 2001), Helsinki, Finland, 11-15 June 2001.

[4] N. C. Ericsson, "Adaptive modulation and scheduling of IP traffic over fading channels," Proc. IEEE Veh. Technol. Conf. (VTC '99 Fall), Amsterdam, The Netherlands, 19-22 Sept 1999.

[5] A. J. Goldsmith, and S.-G. Chua, "Variable-rate variable-power MQAM for fading channels," IEEE Trans. Commun., vol. 45, no. 10, pp. 12181230, Oct 1997

[6] S. Vishwanath, and A. Goldsmith, "Adaptive turbo-coded modulation for flat-fading channels," IEEE Trans. Commun., vol. 51, no. 6, pp. 964-972, June 2003.

[7] H. S. Wang, and N. Moayeri, "Finite-state Markov channel - A useful model for radio communication channels," IEEE Trans. Veh. Technol., vol. 44, no. 1, pp. 163-171, Feb 1995.

[8] V. Tralli, and M. Zorzi, "Markov models for the physical layer block error process in a WCDMA cellular system," Proc. IEEE Global Telecommun. Conf. (GLOBECOM 2002), Taipei, Taiwan, 17-21 Nov 2002.

[9] U. Celentano, and S. Glisic, "Characterization of the link layer service capacity of adaptive air interfaces with imperfections," IEEE Trans. Wireless Commun., Submitted.

[10] L. Hanzo, C. H. Wong, and M. S. Yee, Adaptive wireless transceivers, Chichester, England: John Wiley \& Sons, 2002, pp. 191-255.

[11] Y. Y. Kim, and S.-Q. Li, "Capturing important statistics of a fading/shadowing channel for network performance analysis," IEEE $J$. Select. Areas Commun., vol. 17, no. 5, pp. 888-901, May 1999. 